\title{
Nicotinamide mononucleotide redox cofactor system enables aldehyde accumulation in Escherichia coli
}

Kelly N. Richardson ${ }^{1, a}$, William B. Black ${ }^{1, a}$, and Han $\mathrm{Li}^{1^{*}}$

${ }^{1}$ Department of Chemical and Biomolecular Engineering, University of California Irvine, ${ }^{\text {a These authors }}$ contributed equally. *To whom correspondence should be addressed

\begin{abstract}
It is challenging to biosynthesize industrially important aldehydes, which are readily consumed by the numerous alcohol dehydrogenases (ADHs) in cells. In this work, we demonstrate that a nicotinamide mononucleotide $\left(\mathrm{NMN}^{+}\right)$-dependent redox cofactor cycling system enables aldehyde accumulation in Escherichia coli crude lysates and whole cells. By specifically delivering reducing power to a recombinant enoate reductase, but not to endogenous ADHs, we convert citral to citronellal with minimal byproduct formation (98\% and $83 \%$ product purity in crude lysate- and whole cell-based biotransformation, respectively). We envision the system's universal application to lower the noise in biomanufacturing by silencing the host's metabolic background.
\end{abstract}

\section{KEYWORDS}

Nicotinamide mononucleotide, aldehyde, citronellal, noncanonical redox cofactor, Escherichia coli, biomimetic cofactor

\section{Introduction}

Controlling the flow of mass and energy through a cell's metabolism is a central theme of biocatalyst development. Limiting side reactions is key to developing processes that are efficient and economically viable in the increasingly petroleum-dependent world ${ }^{1,2}$. The need for controlling side reactions is particularly evident in the biorenewable production of aldehydes, which are used industrially for flavors, odorants, and as a precursor to plastics and resins $\mathrm{s}^{3-5}$. Engineering microorganisms to accumulate aldehydes has proven difficult due to the high activity and broad substrate ranges of a myriad of $\mathrm{NAD}(\mathrm{P}) \mathrm{H}$-dependent alcohol dehydrogenases (ADHs) in the cell, which readily reduce aldehydes to alcohols $^{6-9}$.

To overcome this challenge, various techniques have been employed. In Saccharomyces cerevisiae, pathway modules have been compartmentalized in organelles to physically isolate the accumulating aldehydes from competing enzymes ${ }^{10,11}$. In Escherichia coli, screening, characterization, and disruption of aldehyde reductases and ADHs has enabled the engineering of several aldehyde accumulating strains ${ }^{3,12}$. For example, Rodriguez and coworkers generated an aliphatic aldehyde accumulating strain by screening 44 candidate aldehyde reductases and ultimately identifying and disrupting 13 genes $^{3}$. Similarly, Kunjapur and coworkers developed an aromatic aldehyde accumulating E. coli strain, termed reduced aromatic aldehyde reduction (RARE) strain, by disrupting six aldehyde reductases ${ }^{12}$. They then used the RARE strain in the production of benzaldehyde and vanillin ${ }^{12}$. While both aldehyde accumulating strains enabled significantly increased aldehyde titer and yields, success of these strains is substrate-dependent ${ }^{13,14}$, and both groups have recognized that uncharacterized consumption of aldehydes still persist ${ }^{3,12}$. Furthermore, the process of identifying, characterizing, and screening the numerous reductases is time consuming and labor intensive, a task which becomes even more arduous when engineering non-model organisms without well understood metabolisms. 
Recently, we demonstrated the use of a nicotinamide mononucleotide $\left(\mathrm{NMN}^{+}\right)$-dependent orthogonal redox cofactor system to control pathway flux without the need to genetically disrupt competing pathways ${ }^{15}$. Here, we demonstrate that this system can serve as a new tool for aldehyde production by curtailing electron supply to ADHs. This concept was previously explored by Paul and coworkers where they employed chemically reduced biomimetic cofactor to decrease ADH activity during in vitro production of citronellal ${ }^{16}$, a terpenoid aldehyde with wide usage in the flavor and fragrance industries, as the precursor of nonracemic menthol, and as an insect repellant ${ }^{17,18}$. However, because this system did not utilize a method of cofactor regeneration, an equimolar supply of chemically reduced cofactor was required. Moreover, its application to in vivo biosynthesis was not achieved.

In this work, we first demonstrate that the $\mathrm{NMN}^{+}$-based orthogonal redox cofactor system can enable citronellal production in crude lysate-based, cell-free biotransformation without the need to identify or disrupt competing ADHs in E. coli. Next, we used the modular crude lysate system to rapidly prototype and optimize the process, resulting in a productivity of $76 \mathrm{mg} / \mathrm{L}$ citronellal in 1 hour, with a product purity to $98 \%$. Finally, the optimized crude lysate system was used to inform whole-cell biotransformation in $E$. coli, which produced $33 \mathrm{mg} / \mathrm{L}$ citronellal with $83 \%$ product purity. In contrast, biotransformation processes relying on the natural cofactors nicotinamide adenine dinucleotide (phosphate) $\left(\mathrm{NAD}(\mathrm{P})^{+}\right)$only yielded the alcohol byproducts, namely citronellol, nerol, and geraniol. We envision this approach may be adapted for different target products and in various organisms with relative ease, since the successful application of the orthogonal redox cofactor technology does not depend on comprehensive knowledge of the host's endogenous metabolic activity.

\section{Results and Discussion}

\section{Design of the $\mathrm{NMN}^{+}$-dependent citronellal production scheme}

We employed Pseudomonas putida enoate reductase XenA to reduce the activated $\mathrm{C}=\mathrm{C}$ double bond in citral to yield citronellal (Figure 1). The reducing power required by XenA is provided by glucose oxidation, which is catalyzed by Bacillus subtilis glucose dehydrogenase (GDH). Depending on whether the wild type GDH (GDH WT) or engineered GDH (GDH Ortho) is used, NAD(P) ${ }^{+}$or the noncanonical cofactor $\mathrm{NMN}^{+}$can be reduced. While XenA can accept reducing power in the forms of both NADP(H) and reduced $\mathrm{NMN}^{+}(\mathrm{NMNH})$, endogenous $\mathrm{ADHs}$ only accept $\mathrm{NAD}(\mathrm{P}) \mathrm{H}$. As a result, when GDH WT is used, all reactions catalyzed by XenA and ADHs can occur, resulting in the production of not only citronellal, but also alcohol byproducts, namely geraniol, nerol, and citronellol (Figure 1A). On the other hand, we hypothesized that when GDH Ortho is used, $\mathrm{NMN}^{+}$will be the only cycling cofactor which delivers electrons specifically to XenA, yielding citronellal as the predominant product (Figure 1B).
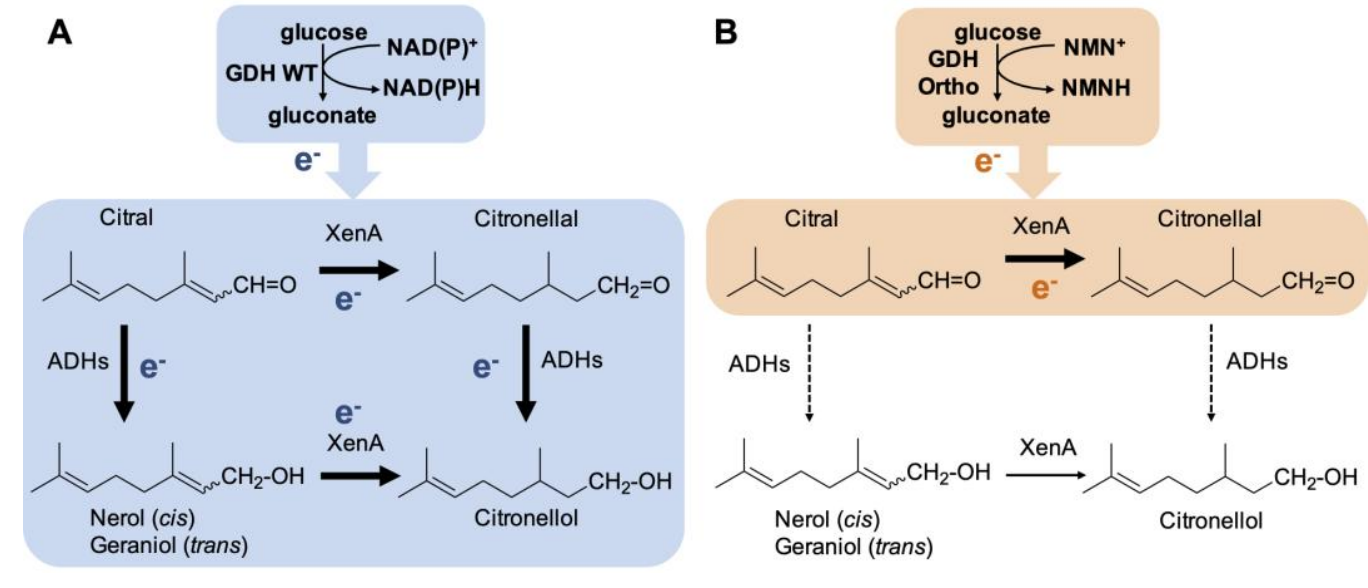
Figure 1: Reaction scheme for citronellal production. A two-part redox cofactor cycling system facilitates the conversion of citral to citronellal through the action of Pseudomonas putida XenA. Redox cofactors are continuously regenerated by the glucose dehydrogenase, GDH, from Bacillus subtilis. (A) When the wild type GDH is supplied, the native cofactors nicotinamide dinucleotide (phosphate), $\mathrm{NAD}(\mathrm{P})^{+}$, are reduced. This supplies the reducing power to both XenA and E. coli 's endogenous alcohol dehydrogenases (ADHs), resulting in the formation of alcohols (nerol, geraniol, citronellol) as the final products. (B) When a nicotinamide mononucleotide $\left(\mathrm{NMN}^{+}\right)$-specific $\mathrm{GDH}, \mathrm{GDH}$ Ortho, is introduced, reducing power is only delivered to XenA, which can readily receive $\mathrm{NMNH}$. Endogenous ADH activity is silenced since $\mathrm{ADH}$ s cannot receive $\mathrm{NMNH}$. Therefore, citronellal, the target product, will accumulate.

\section{Citronellal production in crude lysate-based, cell-free biotransformation}

We first tested our hypothesis in crude lysate-based biotransformation. Crude lysate-based biotransformation serves as a great tool in biocatalysis because it has a cell-like construction and metabolism, but it is freed from the liability of maintaining living cells ${ }^{19-21}$. However, just like in whole cells, controlling pathway flux and limiting non-specific reactions is critical for efficient product formation in crude lysate-based biosynthesis ${ }^{16}$.

The orthogonal redox cofactor system was built in a modular fashion. E. coli crude lysates containing overexpressed different variants of GDH (on plasmids pEK101 or pLZ216, Table S1) or XenA (on plasmid pEK102) were generated separately and subsequently mixed-and-matched to yield a complete cycling system for each experimental condition. The E. coli host, MX102 (Table S1), also contains knockouts to block reducing power generation from glucose by the host's native metabolism ${ }^{15}$. When XenA and GDH WT lysates were mixed at a 1:1 ratio, and with the supplementation of no additional cofactor, $1 \mathrm{mM} \mathrm{NAD}^{+}$, or $1 \mathrm{mM} \mathrm{NADP}^{+}$, citronellal only transiently accumulated to a low level $(<10 \mathrm{mg} / \mathrm{L}$ ) before being rapidly consumed (Figure 2A). Notably, the substrate citral also disappeared rapidly (Figure S1). These results highlight the challenge in citral biotransformation into citronellal since both the substrate and the product are aldehydes.

Importantly, when GDH Ortho was used in place of GDH WT, $89 \mathrm{mg} / \mathrm{L}$ of citronellal was produced with a $39 \%$ product purity (the percentage of citronellal in the sum of all products including citronellal, geraniol, nerol, and citronellol) when $1 \mathrm{mM}$ of $\mathrm{NMN}^{+}$was supplemented (Figure 2A, B). When $\mathrm{NMN}^{+}$was omitted, citronellal production was not significant, demonstrating that aldehyde accumulation was indeed $\mathrm{NMN}^{+}$-dependent (Figure 2A). Remarkably, this was achieved without disrupting any aldehyde reductases or ADHs genes.

A

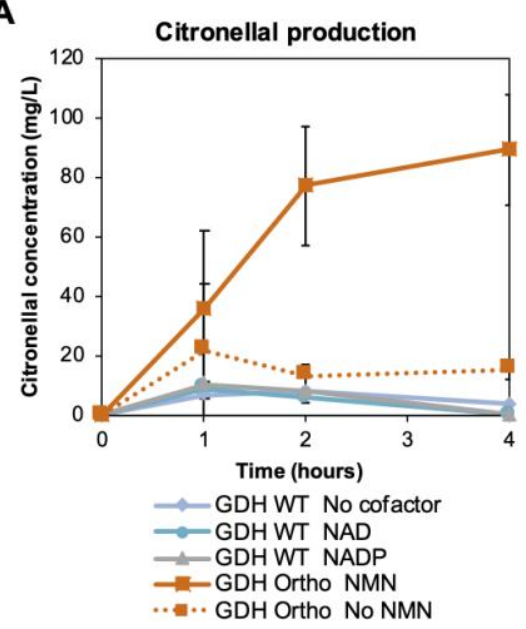

B

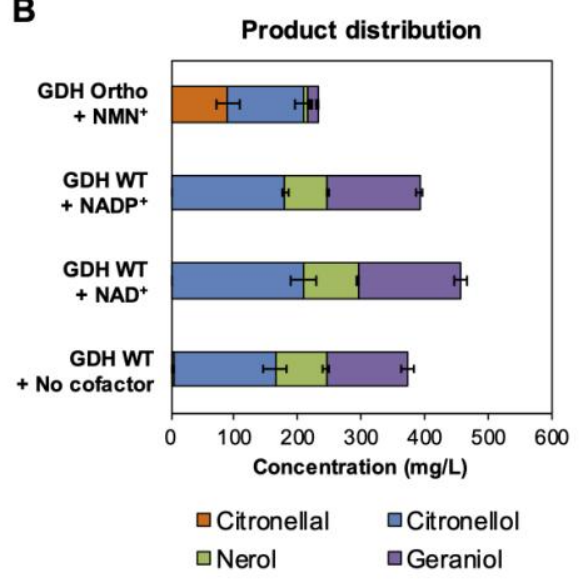


Figure 2: $\mathrm{NMN}^{+}$-dependent orthogonal redox cofactor system enables citronellal accumulation. Crude Escherichia coli lysates enriched with XenA and wild type Bacillus subtilis GDH (GDH WT) or engineered GDH (GDH Ortho) was used to convert citral to citronellal. (A) When GDH WT was used with XenA, citronellal only transiently accumulated to low levels. When GDH Ortho was used with XenA, citronellal was able to accumulate. (B) Product distribution of cycling reactions. The reactions contain 4.5 $\mathrm{mg} / \mathrm{L}$ lysates, $12 \mathrm{mM}$ magnesium acetate, $10 \mathrm{mM}$ ammonium acetate, $130 \mathrm{mM}$ potassium acetate, $10 \mathrm{mM}$ potassium phosphate, $200 \mathrm{mM}$ D-glucose, $1 \mathrm{mM}$ of oxidized cofactor, and $500 \mathrm{mg} / \mathrm{L}$ citral. Reactions were incubated at $37^{\circ} \mathrm{C}$. Values are an average of at least three replicates, and the error bars represent one standard deviation.

\section{Optimizing the crude lysate-based biotransformation}

Although the crude-lysate system was able to accumulate citronellal, approximately $60 \%$ of the products were still alcohols, with the major byproduct being citronellol (119 mg/L) (Figure 2E). We hypothesized that although GDH Ortho specifically generates NMNH from glucose, NAD(P)H is still generated from other substrates present in crude lysates, such as lipids, amino acids, and pyruvate, and that this generation of $\mathrm{NAD}(\mathrm{P}) \mathrm{H}$ was enabling alcohol formation. Although the amount of $\mathrm{NAD}(\mathrm{P}) \mathrm{H}$ generated through these background activities may be small, the highly active, endogenous ADHs are able to use it efficiently. Instead of screening and disrupting the numerous pathways in E. coli that can generate $\mathrm{NAD}(\mathrm{P}) \mathrm{H}$, we sought to increase XenA activity to outcompete ADHs, and to optimize the balance between GDH Ortho and XenA.

We capitalized on the modularity of the system to rapidly prototype various ratios of XenA:GDH Ortho lysates ${ }^{22,23}$, with the total amount of lysates held constant. The 1:1 ratio tested in the initial proofof-concept (Figure 2) only produced $24 \mathrm{mg} / \mathrm{L}$ of citronellal with a product purity of $44 \%$ in 1 hour (Figure $3 \mathrm{~A})$. While increasing the proportion of GDH Ortho had a detrimental effect, shifting the ratio towards XenA resulted in increased citronellal production; this is consistent with our hypothesis that the productivity was impeded by poor relative XenA activity (Figure 3A). The 7:1 (XenA:GDH Ortho) ratio produced $69 \mathrm{mg} / \mathrm{L}$ of citronellal and a $78 \%$ product purity, with $16 \mathrm{mg} / \mathrm{L}$ citronellol (Figure 3A) and undetectable levels of geraniol and nerol (data not shown) produced. Increasing the XenA:GDH Ortho ratio to 11:1 further diminished citronellol production and increased product purity of citronellal to $86 \%$ (Figure 3A). However, this increase in purity came at the loss of citronellal production to $65 \mathrm{mg} / \mathrm{L}$ (Figure 3A), likely because GDH Ortho became limiting in this condition. These results indicated that the cycling system functioned most effectively at a 7:1 XenA:GDH Ortho ratio. When we added an ultracentrifugation step in lysate preparation to further remove interfering enzymes which are associated with the membranes or present in high molecular-weight protein complexes, the productivity and purity was further improved (Figure 3B). When ultracentrifuge lysates were tested at the 7:1 XenA:GDH Ortho ratio, $75 \mathrm{mg} / \mathrm{L}$ citronellal was produced in 1 hour with a $98 \%$ product purity. Alcohol byproduct formation was blocked, with $<2 \mathrm{mg} / \mathrm{L}$ citronellol and undetectable levels of geraniol and nerol produced (Figure 3B). 

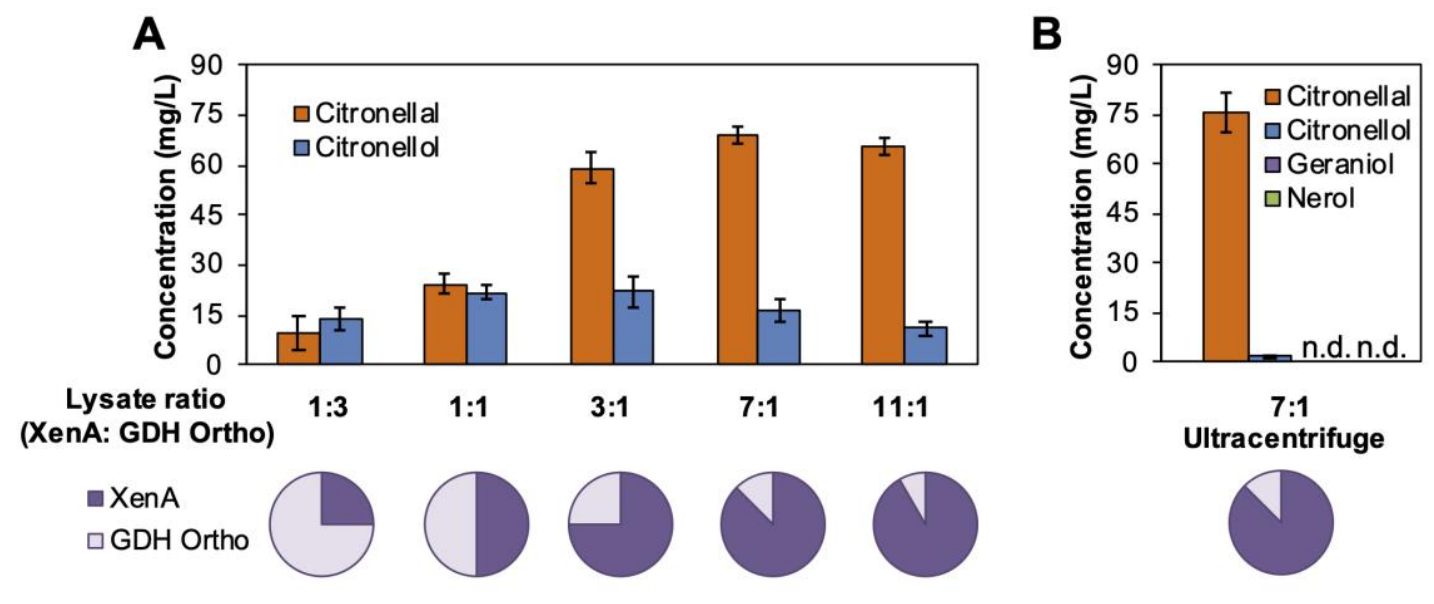

Figure 3: Optimizing crude lysate-based citronellal production. (A) Increasing the relative amount of XenA- to GDH Ortho-enriched E. coli crude lysate enabled increased citronellal production and specificity. (B) Further clarifying the lysate with an ultracentrifuge further improved the performance, yielding a $>98 \%$ pure production of citronellal. The reactions contain $4.5 \mathrm{mg} / \mathrm{L}$ lysates, $200 \mathrm{mM}$ D-glucose, $1 \mathrm{mM}$ of oxidized cofactor, and $500 \mathrm{mg} / \mathrm{L}$ citral and were incubated at $37{ }^{\circ} \mathrm{C}$ for 1 hour. Values are an average of at least three replicates, and the error bars represent one standard deviation. n.d., not detected.

\section{Citronellal production in $E$. coli whole cells}

We next examined the ability of the orthogonal cofactor system to produce citronellal in vivo. Consistent with the cell-free process, when resting E. coli whole cells were supplied with XenA (on plasmid pEK101, Table S1), GDH WT (on plasmid pSM107), and a glucose facilitator, Zymomonas mobilis Glf (on plasmid pSM109) which transports glucose into the cell, endogenous ADH activity completely consumed citral and citronellal, resulting in the production of only geraniol, nerol, and citronellol (Figure 4A). Importantly, when GDH Ortho was used to replace GDH WT, aldehyde accumulation was achieved (Figure 4B).

Previously, crude-lysate based biotransformation has been shown as a powerful tool to prototype and inform pathway design in whole-cells ${ }^{24,25}$. Here, we demonstrated the benefit of maintaining a higher XenA:GDH Ortho ratio is translatable from in vitro to in vivo. We introduced the orthogonal cofactor cycling system using two different configurations (Figure 4B). When high XenA expression was paired with low GDH Ortho expression (on plasmids pEK101 and pSM106, respectively. Figure S2, Table S1), $33 \mathrm{mg} / \mathrm{L}$ of citronellal was produced with a product purity of $83 \%$ (Figure 4B). Conversely, pairing high GDH Ortho expression with low XenA expression (on plasmids pLZ216 and pLZ217, respectively) caused a significant decrease in citronellal production $(6 \mathrm{mg} / \mathrm{L})$, and a poor product purity of $42 \%$. In both conditions, the major byproduct citronellol was formed at $7 \mathrm{mg} / \mathrm{L}$.

The orthogonal redox cofactor system is highly complementary to the commonly used host engineering methods. For example, while the orthogonal redox cofactor system bypassed the laborious process of knocking out the numerous, highly promiscuous ADHs and aldehyde reductases ${ }^{3,12}$, efforts to genetically eliminate the fewer, more substrate specific, and more easily identifiable competing enzymes that consume citral and citronellal (namely aldehyde dehydrogenases and citral lyase) may further improve biotransformation performance ${ }^{17,26}$. 
A

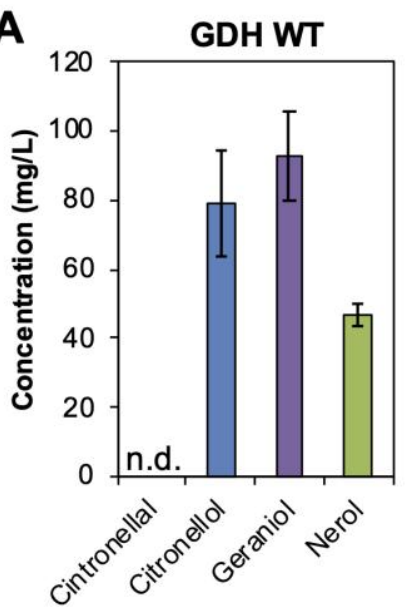

B

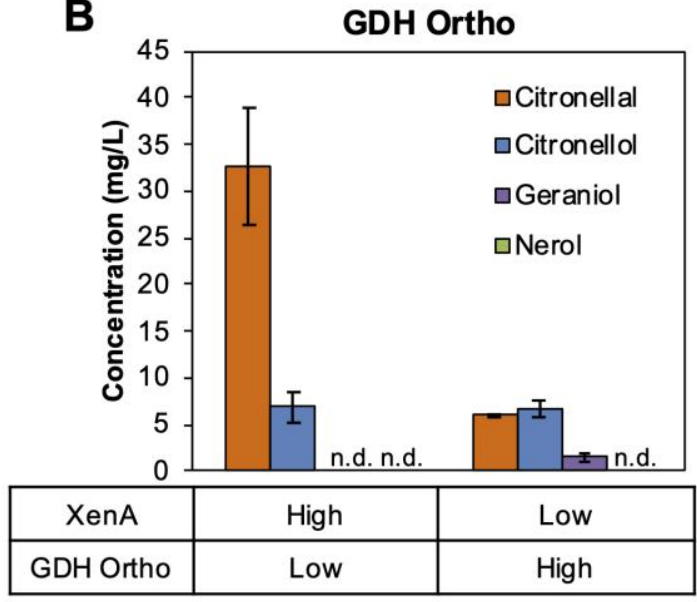

Figure 4: Orthogonal cofactor system enables specific aldehyde accumulation in $E$. coli whole cells. A cofactor cycling system which cycles $\mathrm{NAD}(\mathrm{P})^{+}$(via GDH WT) or $\mathrm{NMN}^{+}$(via GDH Ortho) was introduced to resting $E$. coli cells to enable the conversion of citral to citronellal by XenA. (A) With GDH WT, only alcohol byproducts derived from citral and citronellal were produced. (B) With GDH Ortho, citronellal accumulation was achieved. High XenA expression relatively to GDH Ortho resulted in higher citronellal productivity and product purity. Whole-cell biotransformation was performed with resting $E$. coli cells at an OD600 of 10 with $200 \mathrm{mM} \mathrm{D-glucose,} 2 \mathrm{mM} \mathrm{NMN}^{+}$, and $500 \mathrm{mg} / \mathrm{L}$ citral, at $30{ }^{\circ} \mathrm{C}$ for 3 hours while shaking at 250 r.p.m. Values are an average of at least three replicates, and the error bars represent one standard deviation. n.d., not detected.

\section{Conclusion}

This work demonstrates the use of an $\mathrm{NMN}^{+}$-dependent orthogonal redox cofactor system as a facile method to develop biocatalysts for aldehyde production. As a proof-of-concept, we achieved effective conversion of citral to citronellal (both are aldehydes) by silencing the highly active ADHs in both $E$. coli crude lysates and whole cells. Notably, we decreased the level of alcohol byproducts in crude lysate-based biotransformation from $60 \%$ to $2 \%$, by only optimizing the components in the orthogonal redox loop, and without relying on any information about the host's natural metabolism. The ease in optimization highlights the advantage of an insulated, orthogonal metabolic system. Compared to methods which aim to achieve insulation through physical compartmentalization ${ }^{10,11}$, the orthogonal redox cofactor technology chemically insulates aldehydes from competing ADHs. This avoids the need to target and transport enzymes and chemicals into natural or artificial organelles, and is inherently beneficial in crude-lysate based biotransformation where cells are homogenized and the spatial organization may be lost. As more enzymes are engineered to efficiently utilize $\mathrm{NMN}(\mathrm{H})$, the methods established here may be readily adapted to produce various chemicals and in different microbial chassis.

\section{SUPPORTING INFORMATION}

Experimental methods, plasmids and strains used in this study (Table S1), accession numbers for proteins used in this study (Table S2), citral consumption in crude lysate-based biotransformation (Figure S1), and expression levels of different vectors as measured by enzyme activity in crude lysate (Figure S2).

\section{AUTHOR INFORMATION}




\section{Corresponding Author}

* Han Li (Email: han.li@uci.edu)

\section{AUTHOR CONTRIBUTION}

H.L. conceived the work. K.N.R. and W.B.B. performed the experiments. H.L., K.N.R., and W.B.B. analyzed the data. H.L., K.N.R., and W.B.B. wrote the manuscript.

\section{NOTES}

The authors declare no competing financial interest.

\section{ACKNOWLEDGMENT}

H.L. acknowledges support from University of California, Irvine, the National Science Foundation (NSF) (award no. 1847705), and the National Institutes of Health (NIH) (award no. DP2 GM137427). W.B.B. acknowledges support from Graduate Assistance in Areas of National Need fellowship funded by the U.S. Department of Education. The content is solely the responsibility of the authors and does not necessarily represent the official views of the National Institutes of Health or the NSF.

\section{REFERENCES}

[1] A. M. Kunjapur and K. L. J. Prather, "Microbial Engineering for Aldehyde Synthesis," Applied and Environmental Microbiology, vol. 81, no. 6, pp. 1892-1901, 2015.

[2] C. T. Trinh, P. Unrean and F. Srienc, "Minimal Escherichia coli Cell for the Most Efficient Production of Ethanol from Hexoses and Pentoses," Applied and Environmental Microbiology, vol. 74, no. 12, pp. 3634-3643, 2008.

[3] G. M. Rodriguez and S. Atsumi, "Toward Aldehyde and Alkane Production by Removing Aldehyde Reductase Activity in Escherichia coli," Metabolic Engineering, vol. 25, pp. 227-237, 2014.

[4] E. H. Hansen, B. L. Møller, G. R. Kock, C. M. Bünner, C. Kristensen, O. R. Jensen, F. T. Okkels, C. E. Olsen, M. S. Motawia and J. Hansen, "De novo Biosynthesis of Vanillin in Fission Yeast (Schizosaccharomyces pombe) and Baker's Yeast (Saccharomyces cerevisiae)," Applied and Environmental Microbiology, vol. 75, no. 9, pp. 2765-2774, 2009.

[5] J. Chabbi, O. Jennah, N. Katir, M. Lahcini, M. Bousmina and A. El Kadib, "Aldehydefunctionalized chitosan-montmorillonite films as dynamically-assembled, switchable-chemical release bioplastics," Carbohydrate Polymers, vol. 183, pp. 297-293, 2018.

[6] L. C. Fardelone, J. Augusto, R. Rodrigues and P. J. S. Moran, "Baker's Yeast Mediated Asymmetric Reduction of Cinnamaldeyhde Derivatives," Journal of Molecular Catalysis B: Enzymatic, vol. 29, pp. 41-45, 2004.

[7] G. M. Rodriguez and S. Atsumi, "Isobutyraldeyhde Production from Escherichia coli by Removing Aldehyde Reductase Activity," Microbial Cell Factories, vol. 11, no. 90, 2012.

[8] L. R. Jarboe, "YqhD: A Broad-Substrate Range Aldehyde Reductase with Various Applications in Production of Biorenewable Fuels and Chemicals," Applied Microbiology and Biotechnology, vol. 89, pp. 249-257, 2011. 
[9] J. L. Foo, A. V. Susanto, J. D. Keasling, S. S. J. Leong and M. W. Chang, "Whole-cell Biocatalytic and de novo Production of Alkanes from Free Fatty Acids in Saccharomyces cerevisiae," Biotechnology and Bioengineering, vol. 114, pp. 232-237, 2017.

[10] Y. J. Zhou, N. A. Buijs, Z. Zhu, D. O. Gómez, A. Boonsombuti, V. Siewers and J. Nielsen, "Harnessing Yeast Peroxisomes for Biosynthesis of Fatty-Acid-Derived Biofuels and Chemicals with Relieved Side-Pathway Competition," Journal of the American Chemical Society, vol. 138, no. 47, pp. 15368-15377, 2016.

[11] Y. H. Lau, T. W. Giessen and W. Altenburg, "Prokaryotic Nanocompartments Form Synthetic Organelles in a Eukaryote," Nature Communications, vol. 9, no. 1311, 2018.

[12] A. M. Kunjapur, Y. Tarasova and K. L. J. Prather, "Synthesis and Accumulation of Aromatic Aldehydes in an Engineered Strain of Escherichia coli," Journal of the American Chemical Society, vol. 136, no. 33, pp. 11644-11654, 2014.

[13] D. Schwendenwein, G. Fiume, H. Weber, F. Rudroff and M. Winkler, "Selective Enzymatic Transformation to Aldehydes in vivo by Fungal Carboxylate Reductase from Neurospora crassa," Advanced Synthesis \& Catalysis, vol. 358, no. 21, pp. 3414-3421, 2016.

[14] B. Thompson, M. Machas and D. R. Nielsen, "Creating Pathways Towards Aromatic Building Blocks and Fine Chemicals," Current Opinion in Biotechnology, vol. 36, pp. 1-7, 2015.

[15] W. B. Black, L. Zhang, M. W. S, S. Maxel, Y. Cui, E. King, B. Fong, A. Sanchez Martinez, J. B. Siegel and H. Li, "Engineering a Nicotinamide Mononucleotide Redox Cofactor System for Biocatalysis," Nature Chemical Biology, vol. 16, pp. 87-94, 2020.

[16] C. E. Paul, S. Gargiulo, D. J. Opperman, I. Lavandera, V. Gotor-Fernández, V. Gotor, A. Taglieber, I. W. C. E. Arends and F. Hollmann, "Mimicking Nature: Synthetic Nicotinamide Cofactors for $\mathrm{C}=\mathrm{C}$ Bioreduction Using Enaote Reductases," Organic Letters, vol. 15, no. 1, pp. 180-183, 2013.

[17] M. Hall, B. Hauer, R. Stuermer, W. Kroutil and K. Faber, "Asymmetric Whole-Cell Bioreduction of an $\alpha, \beta$-Unsaturated Aldehyde (Citral): Competing prim-Alcohol Dehydrogenase and C-C Lyase Activities," Tetrahedron: Asymmetry, vol. 17, no. 21, pp. 3058-3062, 2006.

[18] V. S. Mahalwal and M. Ali, "Volatile Constituents of Cymbopogon nardus (Linn.) Rendle," Flavour and Fragrance Journal, vol. 18, pp. 73-76, 2003.

[19] W. Wang, M. Liu, C. You, Z. Li and Y. H. P. Zhang, "ATP-Free Biosynthesis of a High-Energy Phosphate Metabolite Fructose 1,6-diphosphate by in vitro Metabolic Engineering," Metabolic Engineering, vol. 42, pp. 168-174, 2017.

[20] K. Honda, K. Kimura, P. H. Ninh, H. Taniguchi, K. Okano and H. Ohtake, "In vitro Bioconversion of Chitin to Pyruvate with Thermophilic Enzymes," Journal of Bioscience and Bioengineering, vol. 124, no. 3, pp. 296-301, 2017.

[21] J. E. Kay and M. C. Jewett, "A Cell-Free System for Production of 2,3-Butanediol is Robust to Growth-Toxic Compounds," Metabolic Engineering Communications, vol. 10, 2020. 
[22] Q. M. Dudley, K. C. Anderson and M. C. Jewett, "Cell-Free Mixing of Escherichia coli Crude Extracts to Prototype and Rationally Engineer High-Titer Mevalonate Synthesis," ACS Synthetic Biology, vol. 5, no. 12, pp. 1578-1588, 2016.

[23] Z. Liu, Y. Zhang, X. Jia, M. Hu, Z. Deng, Y. Xu and T. Liu, "In Vitro Reconstitution and Optimization of the Entire Pathway to Convert Glucose to Fatty Acid," ACS Synthetic Biology, vol. 6, no. 4, pp. 701-709, 2017.

[24] A. S. Karim and M. C. Jewett, "A Cell-Free Framework for Rapid Biosynthetic Pathway Prototyping and Enzyme Discovery," Metabolic Engineering, vol. 36, pp. 116-126, 2016.

[25] R. Kelwick, L. Ricci, S. M. Chee, D. Bell, A. J. Webb and P. S. Freemont, "Cell-Free Prototyping Strategies for Enhancing the Sustainable Production of Polyhdroxyalkanoates Bioplastics," Synthetic Biology, vol. 3, no. 1, 2018.

[26] A. Esmaeili, S. Rohany and S. Safaiyan, "Biotransformation of Citral by Free and Immobilized Saccharomyces cerevisiae," Chemistry of Natural Compounds, vol. 48, pp. 322-324, 2012. 